\title{
The Cyclic Groups via Bezout Matrices
}

\section{Ömür Deveci ${ }^{1}$, Yeşim Aküzüm ${ }^{1}$, Erdal Karaduman ${ }^{2} \&$ Özgür Erdağ ${ }^{1}$}

${ }^{1}$ Kafkas University, Department of Mathematics Faculty of Science and Letters, 36100 Kars, Turkey

${ }^{2}$ Department of Mathematics, Faculty of Science, Atatürk University, 25240 Erzurum, Turkey

Correspondence: Ömür Deveci, Kafkas University, Department of Mathematics Faculty of Science and Letters, 36100 Kars, Turkey. E-mail: odeveci36@hotmail.com

Received: February 25, 2015 Accepted: March 11, 2015 Online Published: March 22, 2015

doi:10.5539/jmr.v7n2p34 URL: http://dx.doi.org/10.5539/jmr.v7n2p34

\begin{abstract}
In this paper, we define the Bezout matrices by the aid of the characteristic polynomials of the $k$-step Fibonacci, the generalized order- $k$ Pell and the generalized order- $k$ Jacobsthal sequences then we consider the multiplicative orders of the Bezout matrices when read modulo $m$. Consequently, we obtain the rules for the order of the cyclic groups by reducing the Bezout matrices modulo $m$.
\end{abstract}

Keywords: Bezout Matrix, cyclic group, order

2010 Mathematics Subject Classification: 15B36, 15A15, 20H20, 20Kxx,

\section{Introduction and Preliminaries}

Let $D$ be an integral domain and $P(x), Q(x) \in D[x]$ with $\operatorname{deg}(P(x))=n$ and $\operatorname{deg}(Q(x))=m$, we assume $n \geq m$,

$$
\begin{gathered}
P(x)=u_{n} x^{n}+u_{n-1} x^{n-1}+\cdots+u_{1} x+u_{0}, \\
Q(x)=v_{m} x^{m}+v_{m-1} x^{m-1}+\cdots+v_{1} x+v_{0} .
\end{gathered}
$$

The Bezout matrix associated to the polynomials $P(x)$ and $Q(x)$ is the symmetric matrix:

$$
B_{n}(P, Q)=\left[b_{i j}\right]_{n \times n}
$$

where the entries $b_{i j}$ are obtained by the identity

$$
\frac{P(x) Q(y)-P(y) Q(x)}{x-y}=\sum_{i, j=1}^{n} b_{i j} x^{i} y^{j} .
$$

It is important to note that the Bezout matrix $B_{n}(P, Q)$ is in $D^{n \times n}$ and the entries $b_{i j}$ are defined by the formula

$$
b_{i j}=\sum_{k=1}^{m_{i j}} u_{i+k-1} v_{i-k}-u_{i-k} v_{j+k-1}
$$

such that $m_{i j}=\min \{i, n+1-j\}$ for each $i, j=1,2, \cdots, n$.

For more information on the Bezout matrix, see (Cayley, 1857; Barnett, 1972; Householder, 1970; Sylwester, 


\section{3).}

The $k$-step Fibonacci sequence $\left\{F_{n}^{k}\right\}$ is defined recursively by the equation

$$
F_{n+k}^{k}=F_{n+k-1}^{k}+F_{n+k-2}^{k}+\cdots+F_{n}^{k}
$$

for $n \geq 0$, where $F_{0}^{k}=F_{1}^{k}=F_{k-2}^{k}=0$ and $F_{k-1}^{k}=1$.

For more information on the $k$-step Fibonacci sequence $\left\{F_{n}^{k}\right\}$, see (Kalman, 1982; Slone).

In (Kilic \& Tasci, 2006), Kilic and Tasci defined the generalized order- $k$ Pell sequence $\left\{P_{n}^{k}\right\}$ as follows:

For $n>0$,

$$
P_{n}^{k}=2 P_{n-1}^{k}+P_{n-2}^{k}+\cdots+P_{n-k}^{k}
$$

with initial conditions $P_{1-k}^{k}=1$ and $P_{2-k}^{k}, \cdots, P_{0}^{k}=0$.

The generalized order- $k$ Jacobsthal sequence $\left\{J_{n}^{k}\right\}$ is defined (Yilmaz \& Bozkurt, 2009) recursively by the equation

$$
J_{n}^{k}=J_{n-1}^{k}+2 J_{n-2}^{k}+\cdots+J_{n-k}^{k}
$$

for $n>0$, where $J_{1-k}^{k}=1$ and $J_{2-k}^{k}, \cdots, J_{0}^{k}=0$.

In (Deveci \& Akuzum, 2014; Deveci \& Karaduman, 2012; Deveci \& Karaduman, in press; Deveci, et al., in press; Lü \& Wang, 2007; Ozkan, 2014; Tas, et al., 2014; Tas \& Karaduman, 2014), the authors obtained the cyclic groups via some special matrices. In this paper, we define the Bezout matrices by the aid of the characteristic polynomials of the $k$-step Fibonacci, the generalized order- $k$ Pell and the generalized order- $k$ Jacobsthal sequences. Further, we consider the multiplicative orders of the Bezout matrices according to modulo $m$ and so we obtain the rules for the orders of the cyclic groups which are produced using the Bezout matrices as generators by reducing their elements according to modulo $m$.

\section{Main Results and Proofs}

It is easy to see that the characteristic polynomials of the $k$-step Fibonacci, the generalized order- $k$ Pell and the generalized order- $k$ Jacobsthal sequences are as follows, respectively:

$$
\begin{gathered}
P_{k}^{F}(x)=x^{k}-x^{k-1}-\cdots-x-1, \\
P_{k}^{P}(x)=x^{k}-2 x^{k-1}-x^{k-2}-\cdots-x-1
\end{gathered}
$$

and

$$
P_{k}^{J}(x)=x^{k}-x^{k-1}-2 x^{k-2}-x^{k-3}-\cdots-1
$$

Then we can write the following Bezout matrices for the polynomials $P_{k}^{F}(x), P_{k}^{P}(x)$ and $P_{k}^{J}(x)$.

Definition 2.1. For every positive integer $k \geq 3$, the Bezout matrix $B_{k}\left(P_{k}^{F}(x), P_{k-1}^{F}(x)\right)=\left[b_{i j}\right]_{k \times k}$ is as follows: 


$$
b_{i(k-t)}=\left\{\begin{aligned}
0, & i<t<k, \\
2, & i=t<k, \\
1, & (0<t<i<k) ;(t=0 \text { and } i=k), \\
-1, & (t=0 \text { and } i<k) ;(t<k \text { and } i=k) .
\end{aligned}\right.
$$

That is,

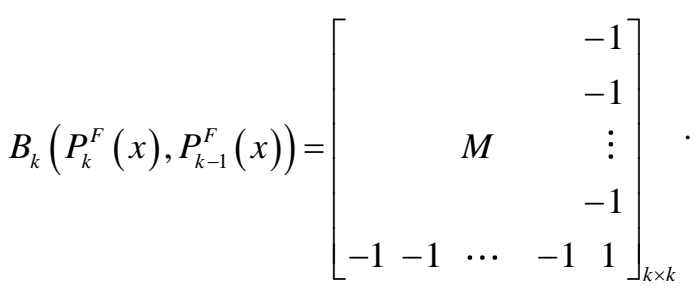

where $M$ is a square matrix of order $k-1$ such that

$$
M=\left[\begin{array}{lllll}
0 & \cdots & 0 & 0 & 2 \\
0 & \cdots & 0 & 2 & 1 \\
\vdots & \therefore & . & \ddots & \vdots \\
0 & 2 & 1 & \cdots & 1 \\
2 & 1 & 1 & \cdots & 1
\end{array}\right]_{(k-1) \times(k-1)}
$$

\section{Example.}

$$
B_{5}\left(P_{5}^{F}(x), P_{4}^{F}(x)\right)=\left[\begin{array}{rrrrr}
0 & 0 & 0 & 2 & -1 \\
0 & 0 & 2 & 1 & -1 \\
0 & 2 & 1 & 1 & -1 \\
2 & 1 & 1 & 1 & -1 \\
-1 & -1 & -1 & -1 & 1
\end{array}\right]
$$

Definition 2.2. For every positive integer $k \geq 3$, the Bezout matrices $B_{k}\left(P_{k}^{P}(x), P_{k-1}^{P}(x)\right)=\left[b_{i j}\right]_{k \times k}$ are as follows:

If $k=3$, The Bezout matrix $B_{3}\left(P_{3}^{P}(x), P_{2}^{P}(x)\right)$ is

$$
\left[\begin{array}{rrr}
-1 & 3 & -1 \\
3 & 4 & -2 \\
-1 & -2 & 1
\end{array}\right] .
$$

If $k=4$, The Bezout matrix $B_{4}\left(P_{4}^{P}(x), P_{3}^{P}(x)\right)$ is

$$
\left[\begin{array}{rrrr}
0 & -1 & 3 & -1 \\
-1 & 2 & 2 & -1 \\
3 & 2 & 4 & -2 \\
-1 & -1 & -2 & 1
\end{array}\right]
$$


Let $k \geq 5$, then the Bezout matrices $B_{k}\left(P_{k}^{P}(x), P_{k-1}^{P}(x)\right)=\left[b_{i j}\right]_{k \times k}$ are defined by the following form:

$$
b_{i(k-t-1)}=\left\{\begin{aligned}
0, & 1 \leq i<t<k-1, \\
-1, & (1 \leq i=t<k-1) ;(1 \leq i<k-1 \text { and } t=-1) ;(i=k \text { and } 1 \leq t<k-1) \\
2, & (2 \leq i<k-1 \text { and } t=i-1 \text { or } t=0) ;(i=k-1 \text { and } 2 \leq t \leq k-2) \\
-2, & (i=k-1 \text { and } t=-1) ;(i=k \text { and } t=0) \\
3, & (i=1 \text { and } t=0) ;(i=k-1 \text { and } t=k-2) \\
4, & i=k-1 \text { and } t=0 \\
1, & \text { otherwise. }
\end{aligned}\right.
$$

That is,

$$
B_{k}\left(P_{k}^{P}(x), P_{k-1}^{P}(x)\right)=\left[\begin{array}{rrrrrr} 
& & & & 3 & -1 \\
& & & & 2 & -1 \\
& & M & & \vdots & \vdots \\
& & & & 2 & -1 \\
3 & 2 & \cdots & 2 & 4 & -2 \\
-1 & -1 & \cdots & -1 & -2 & 1
\end{array}\right]_{k \times k}
$$

for $k \geq 5$. Where $M$ is a square matrix of order $k-2$ such that

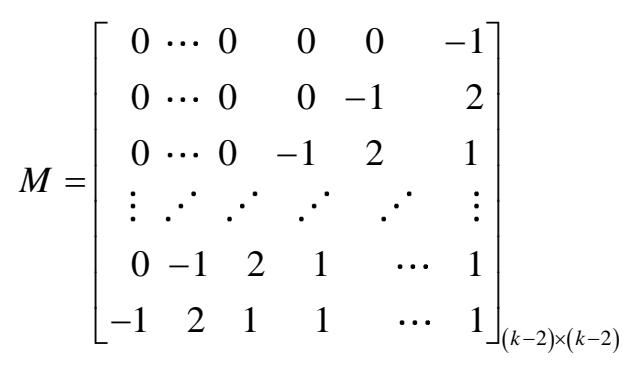

\section{Example.}

$$
B_{6}\left(P_{6}^{P}(x), P_{5}^{P}(x)\right)=\left[\begin{array}{rrrrrr}
0 & 0 & 0 & -1 & 3 & -1 \\
0 & 0 & -1 & 2 & 2 & -1 \\
0 & -1 & 2 & 1 & 2 & -1 \\
-1 & 2 & 1 & 1 & 2 & -1 \\
3 & 2 & 2 & 2 & 4 & -2 \\
-1 & -1 & -1 & -1 & -2 & 1
\end{array}\right] \text {. }
$$

We easily derive that

$$
\operatorname{det} B_{k}\left(P_{k}^{F}(x), P_{k-1}^{F}(x)\right)=\operatorname{det} B_{k}\left(P_{k}^{P}(x), P_{k-1}^{P}(x)\right)=\left\{\begin{array}{c}
-1 \text { if } k \equiv 0,3 \bmod 4, \\
1, \text { otherwise, }
\end{array} \text { for } k \geq 3\right.
$$

Definition 2.3. For every positive integer $k \geq 3$, the Bezout matrices $B_{k}\left(P_{k}^{J}(x), P_{k-1}^{J}(x)\right)=\left[b_{i j}\right]_{k \times k}$ are as follows: 
If $k=3$, The Bezout matrix $B_{3}\left(P_{3}^{J}(x), P_{2}^{J}(x)\right)$ is

$$
\left[\begin{array}{rrr}
3 & 3 & -2 \\
3 & 1 & -1 \\
-2 & -1 & 1
\end{array}\right]
$$

If $k=4$, The Bezout matrix $B_{4}\left(P_{4}^{J}(x), P_{3}^{J}(x)\right)$ is

$$
\left[\begin{array}{rrrr}
0 & 3 & 3 & -2 \\
3 & 6 & 1 & -2 \\
3 & 1 & 1 & -1 \\
-2 & -2 & -1 & 1
\end{array}\right]
$$

Let $k \geq 5$, then the Bezout matrices $B_{k}\left(P_{k}^{J}(x), P_{k-1}^{J}(x)\right)=\left[b_{i j}\right]_{k \times k}$ are defined by the following form:

$$
b_{i(k-t-1)}=\left\{\begin{aligned}
0, & 1 \leq i<t<k-1, \\
3, & (1 \leq i=t<k-1) ;(i=k-1 \text { and } t=k-2) ;(i=1 \text { and } t=0) \\
6, & 2 \leq i<k-1 \text { and } t=i-1, \\
1, & (2 \leq i<k-1 \text { and } t=i-1 \text { or } t=0) ;(i=k \text { and } t=-1) \\
-1, & (i=k-1 \text { and } t=-1) ;(i=k \text { and } t=0) \\
-2, & (1 \leq i<k-1 \text { and } t=-1) ;(i=k \text { and } 1 \leq t<k-1) \\
4, & \text { otherwise. }
\end{aligned}\right.
$$

That is,

$$
B_{k}\left(P_{k}^{P}(x), P_{k-1}^{P}(x)\right)=\left[\begin{array}{rrrrrrc} 
& & & & 3 & -2 \\
& & & & 1 & -2 \\
& & M & & \vdots & \vdots \\
& & & & 1 & -2 \\
3 & 1 & \cdots & 1 & 1 & -1 \\
-2 & -2 & \cdots & -2 & -1 & 1
\end{array}\right]_{k \times k}
$$

for $k \geq 5$. Where $M$ is a square matrix of order $k-2$ such that

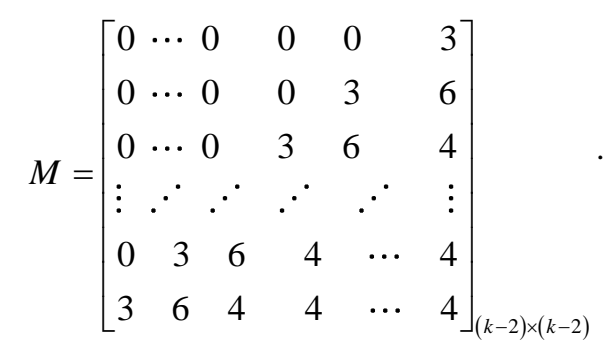

For given a matrix $A=\left[a_{i j}\right]$ with $a_{i j}$ 's being integers, $A(\bmod m)$ means that every entries of $A$ are reduced modulo $m$, that is, $A(\bmod m)=\left(a_{i j}(\bmod m)\right)$. Let $\langle A\rangle_{m}=\left\{(A)^{n}(\bmod m) \mid n \geq 0\right\} . \quad$ If 
$(\operatorname{det} A, m)=1,\langle A\rangle_{m}$ is a cyclic group. We denote the order of the set $\langle A\rangle_{m}$ by $\left|\langle A\rangle_{m}\right|$.

Since $\operatorname{det} B_{k}\left(P_{k}^{F}(x), P_{k-1}^{F}(x)\right)=\operatorname{det} B_{k}\left(P_{k}^{P}(x), P_{k-1}^{P}(x)\right)= \pm 1 \quad$ for $k \geq 3$, it is clear that the sets $\left\langle B_{k}\left(P_{k}^{F}(x), P_{k-1}^{F}(x)\right)\right\rangle_{m}$ and $\left\langle B_{k}\left(P_{k}^{P}(x), P_{k-1}^{P}(x)\right)\right\rangle_{m}$ are cyclic groups for $m \geq 2$.

Now we consider the cyclic groups which are generated by the matrices $B_{k}\left(P_{k}^{F}(x), P_{k-1}^{F}(x)\right)$, $B_{k}\left(P_{k}^{P}(x), P_{k-1}^{P}(x)\right)$ and $B_{k}\left(P_{k}^{J}(x), P_{k-1}^{J}(x)\right)$.

Theorem 2.1. Let $M$ be any of the matrices $B_{k}\left(P_{k}^{F}(x), P_{k-1}^{F}(x)\right), \quad B_{k}\left(P_{k}^{P}(x), P_{k-1}^{P}(x)\right)$ and $B_{k}\left(P_{k}^{J}(x), P_{k-1}^{J}(x)\right)$. Suppose that $\alpha$ is the largest positive integer and $p$ is a prime such that $(\operatorname{det} M, p)=1$ and $\left|\langle M\rangle_{p}\right|=\left|\langle M\rangle_{p^{\alpha}}\right|$. Then $\left|\langle M\rangle_{p^{\lambda}}\right|=p^{\lambda-\alpha} \cdot\left|\langle M\rangle_{p}\right|$ for every $\lambda \geq \alpha$.

Proof. Let us consider the cyclic group $\left\langle B_{k}\left(P_{k}^{F}(x), P_{k-1}^{F}(x)\right)\right\rangle_{m}$ for $k \geq 3$ and $m \geq 2$. Suppose that $a$ is a positive integer and $\left\langle B_{k}\left(P_{k}^{F}(x), P_{k-1}^{F}(x)\right)\right\rangle_{m} \quad$ is denoted by $\quad O(m) \quad$. If $\left(B_{k}\left(P_{k}^{F}(x), P_{k-1}^{F}(x)\right)\right)^{O\left(p^{a+1}\right)} \equiv I\left(\bmod p^{a+1}\right)$, then $\left(B_{k}\left(P_{k}^{F}(x), P_{k-1}^{F}(x)\right)\right)^{O\left(p^{a+1}\right)} \equiv I\left(\bmod p^{a}\right)$ where $I \quad$ is the $k \times k$ identity matrix. Thus we obtain that $O\left(p^{a}\right)$ divides $O\left(p^{a+1}\right)$. Also, writing $\left(B_{k}\left(P_{k}^{F}(x), P_{k-1}^{F}(x)\right)\right)^{O\left(p^{a+1}\right)}=I+\left(b_{i j}^{(a)} \cdot p^{a}\right)$, by the binomial theorem, we obtain

$$
\left(B_{k}\left(P_{k}^{F}(x), P_{k-1}^{F}(x)\right)\right)^{O\left(p^{a+1}\right) \cdot p}=\left(I+\left(b_{i j}^{(a)} \cdot p^{a}\right)\right)^{p}=\sum_{i=0}^{p}\left(\begin{array}{c}
p \\
i
\end{array}\right)\left(b_{i j}^{(a)} \cdot p^{a}\right)^{i} \equiv I\left(\bmod p^{a+1}\right),
$$

which yields that $O\left(p^{a+1}\right)$ divides $O\left(p^{a}\right) \cdot p$. Thus, $O\left(p^{a+1}\right)=O\left(p^{a}\right)$ or $O\left(p^{a+1}\right)=O\left(p^{a}\right) \cdot p$. It is clear that $O\left(p^{a+1}\right)=O\left(p^{a}\right) \cdot p$ holds if and only if there is a $b_{i j}^{(a)}$ which is not divisible by $p$. Since $\alpha$ is the largest positive integer such that $O(p)=O\left(p^{\alpha}\right), O\left(p^{u}\right) \neq O\left(p^{\alpha+1}\right)$. There is an $b_{i j}^{(\alpha+1)}$ which is not divisible by $p$. So we get that $O\left(p^{\alpha+1}\right) \neq O\left(p^{\alpha+2}\right)$. The proof is completed by induction on $\alpha$.

The proofs for the cyclic groups $\left\langle B_{k}\left(P_{k}^{P}(x), P_{k-1}^{P}(x)\right)\right\rangle_{m}$ and $\left\langle B_{k}\left(P_{k}^{J}(x), P_{k-1}^{J}(x)\right)\right\rangle_{m}$ are similar to the above and are omitted.

Example.i. $\left|\left\langle B_{5}\left(P_{5}^{F}(x), P_{4}^{F}(x)\right)\right\rangle_{7}\right|=48$ and so $\left|\left\langle B_{5}\left(P_{5}^{F}(x), P_{4}^{F}(x)\right)\right\rangle_{7^{10}}\right|=1936973136=7^{9} \cdot 48$. 
ii. $\left|\left\langle B_{3}\left(P_{3}^{P}(x), P_{2}^{P}(x)\right)\right\rangle_{11}\right|=12$ and so $\left|\left\langle B_{3}\left(P_{3}^{P}(x), P_{2}^{P}(x)\right)\right\rangle_{11^{20}}\right|=733909085380974555492=11^{19} \cdot 12$.

iii. $\left|\left\langle B_{4}\left(P_{4}^{J}(x), P_{3}^{J}(x)\right)\right\rangle_{5}\right|=104$ and so $\left|\left\langle B_{4}\left(P_{4}^{J}(x), P_{3}^{J}(x)\right)\right\rangle_{5^{15}}\right|=634765625000=5^{14} \cdot 104$.

Theorem 2.2. Let $G_{m}$ be any of the cyclic groups $\left\langle B_{k}\left(P_{k}^{F}(x), P_{k-1}^{F}(x)\right)\right\rangle_{m},\left\langle B_{k}\left(P_{k}^{P}(x), P_{k-1}^{P}(x)\right)\right\rangle_{m}$ and $\left\langle B_{k}\left(P_{k}^{J}(x), P_{k-1}^{J}(x)\right)\right\rangle_{m} \quad$ and $\quad$ let $\quad m=\prod_{n=1}^{t} p_{n}^{e_{n}},(t \geq 1) \quad$ where $\quad p_{i} \quad$ 's $\quad$ are distinct primes, then $\left|G_{m}\right|=\operatorname{Icm}\left[G_{p_{1}^{q_{1}}}, G_{p_{2}^{e_{2}}}, \cdots, G_{p_{k}^{e_{k}}}\right]$.

Proof. Let us consider the cyclic group $B_{k}\left(P_{k}^{J}(x), P_{k-1}^{J}(x)\right)$, then $2 \nmid m$. Let $\left|\left\langle B_{k}\left(P_{k}^{J}(x), P_{k-1}^{J}(x)\right)\right\rangle_{p_{n}^{e_{n}}}\right|=u_{n}$ for $1 \leq i \leq t$ and let $\left|\left\langle B_{k}\left(P_{k}^{J}(x), P_{k-1}^{J}(x)\right)\right\rangle_{m}\right|=u$. Then we have

$$
\text { the entry }(i, j) \text { of }\left(B_{k}\left(P_{k}^{J}(x), P_{k-1}^{J}(x)\right)\right)^{u_{n}}= \begin{cases}p_{n}^{e_{n}} \varepsilon_{i j}, & i>j, \\ p_{n}^{e_{n}} \varepsilon_{i j}+1, & i=j, \\ p_{n}^{e_{n}} \varepsilon_{i j}, & i<j,\end{cases}
$$

and

$$
\text { the entry }(i, j) \text { of }\left(B_{k}\left(P_{k}^{J}(x), P_{k-1}^{J}(x)\right)\right)^{u}= \begin{cases}m \varepsilon_{i j}^{\prime}, & i>j, \\ m \varepsilon_{i j}^{\prime}+1, & i=j, \\ m \varepsilon_{i j}^{\prime}, & i<j,\end{cases}
$$

where $\varepsilon_{i j}$ and $\varepsilon_{i j}^{\prime}$ are integers for $0 \leq i, j \leq k$. Since $m=c \cdot p_{n}^{e_{n}}$ for $1 \leq n \leq t, u$ is of the form $m=c \cdot u_{n}$. Thus we conclude that $|u|=\operatorname{lcm}\left[u_{1}, u_{2}, \cdots, u_{t}\right]$.

The proofs for the cyclic groups $\left\langle B_{k}\left(P_{k}^{F}(x), P_{k-1}^{F}(x)\right)\right\rangle_{m}$ and $\left\langle B_{k}\left(P_{k}^{P}(x), P_{k-1}^{P}(x)\right)\right\rangle_{m}$ are similar to the above and are omitted.

Example.i. Since $\left|\left\langle B_{5}\left(P_{5}^{F}(x), P_{4}^{F}(x)\right)\right\rangle_{5^{3}}\right|=5^{2} \cdot 78=1950,\left|\left\langle B_{11}\left(P_{11}^{F}(x), P_{10}^{F}(x)\right)\right\rangle_{11}\right|=1330$ and $1375=5^{3} \cdot 11$, $\left|\left\langle B_{5}\left(P_{5}^{F}(x), P_{4}^{F}(x)\right)\right\rangle_{1375}\right|=259350=\operatorname{lcm}[1950,1330]$.

ii. Since $\left|\left\langle B_{3}\left(P_{3}^{P}(x), P_{2}^{P}(x)\right)\right\rangle_{2^{5}}\right|=2^{4} \cdot 7=112,\left|\left\langle B_{3}\left(P_{3}^{P}(x), P_{2}^{P}(x)\right)\right\rangle_{3^{4}}\right|=3^{3} \cdot 26=702$ and $2592=2^{5} \cdot 3^{4}$, $\left|\left\langle B_{3}\left(P_{3}^{P}(x), P_{2}^{P}(x)\right)\right\rangle_{2592}\right|=39312=\operatorname{lcm}[112,702]$.

iii. Since $\left|\left\langle B_{4}\left(P_{4}^{J}(x), P_{3}^{J}(x)\right)\right\rangle_{3}\right|=20, \quad\left|\left\langle B_{4}\left(P_{4}^{J}(x), P_{3}^{J}(x)\right)\right\rangle_{5}\right|=104, \quad\left|\left\langle B_{4}\left(P_{4}^{J}(x), P_{3}^{J}(x)\right)\right\rangle_{7}\right|=114 \quad$ and $105=3 \cdot 5 \cdot 7,\left|\left\langle B_{4}\left(P_{4}^{J}(x), P_{3}^{J}(x)\right)\right\rangle_{105}\right|=29640=\operatorname{lcm}[20,104,114]$. 


\section{Conclusion}

Let $M$ be any of the matrices $B_{k}\left(P_{k}^{F}(x), P_{k-1}^{F}(x)\right), B_{k}\left(P_{k}^{P}(x), P_{k-1}^{P}(x)\right)$ and $B_{k}\left(P_{k}^{J}(x), P_{k-1}^{J}(x)\right)$ and let $p \geq k$ be a prime such that $(\operatorname{det} M, p)=1$. Then, we obtain that $\mid\langle M\rangle_{p} \| p^{k+2}-p^{v}$ for $p \leq 2999$ and $0 \leq v \leq k+1$

Open Problem. Is the result above satisfied for every prime $p \geq k$.

\section{Acknowledgment}

This Project was supported by the Commission for the Scientific Research Projects of Kafkas University. The Project number. 2014-FEF-34.

\section{References}

Barnett, S. (1972). A note on the Bezoutian matrix. SIAMJ. Appl. Math., 22(1), 84-86. http://dx.doi.org/10.1137/0122009

Cayley, A. (1857). Note sur la method d'elimination de Bezout. J. Reine Angew. Math., 53, 366-367. http://dx.doi.org/10.1515/crll.1857.53.366

Deveci, O., \& Akuzum, Y. (2014). The cycylic groups via MacWilliams and Chebyshev matrices. Journal of Math. Reseacrh, 6(2), 55-58.

Deveci, O., \& Karaduman, E. (2012). The cyclic groups via the Pascal matrices and the generalized Pascal matrices. Linear Algebra and its Appl., 437, 2538-2545. http://dx.doi.org/10.1016/j.laa.2012.06.024

Deveci, O., \& Karaduman, E., The Pell sequences in finite groups. Util. Math., in Press.

Deveci, O., Akdeniz, M., \& Karaduman, E. The Generalized Pell p-Sequences in Groups. Ars Combinatoria, in Press.

Householder, A. (1970). Householder, Bezoutians, elimination an localization. SIAM Review, 12, 73-78. http://dx.doi.org/10.1137/1012003

Kalman, D. (1982). Generalized Fibonacci numbers by matrix methods. The Fibonacci Quart., 20(1), 73-76.

Kilic, E., \& Tasci, D. (2006). The generalized Binet formula, representation and sums of the generalized order $-k$ Pell numbers. Taiwanese J. Math., 10(6), 1661-1670.

Lü, K., \& Wang, J. (2007). $k$-step Fibonacci sequence modulo m. Util. Math., 71, 169-178.

Ozkan, E. (2014). Truncated Lucas sequences and its period. Appl. Math. and Compt., 232, 285-291. http://dx.doi.org/10.1016/j.amc.2014.01.014

Slone, N. J. A. Sequences A000045/M0692, A000073/M1074, A000078/M1108, A001591, A001622, A046698, A058265, A086088, and A118745 in "The On-Line Encyclopedia of Integer Sequences".

Sylwester, J. J. (1853). On a theory of the syzgetic relations of two rational integral functions, Comrising an application to the theory of Strum's functions, and that of greatest algebraical common measure. Philosophical Transactions of the Royal Society of London, 143, 407-548. http://dx.doi.org/10.1098/rstl.1853.0018

Tas, S., \& Karaduman, E. (2014). The Padovan sequences in finite groups. Chaing Mai J. Sci., 41(2), 456-462.

Tas, S., Deveci, O., \& Karaduman, E. (2014). The Fibonacci-Padovan sequences in finite groups. Maejo Int. J. Sci. and Technol., 8(03), 279-287.

Yilmaz, F., \& Bozkurt, D. (2009). The generalized order-k Jacobsthal numbers. Int. J. Contemp. Math. Sciences, 4(34), 1685-1694.

\section{Copyrights}

Copyright for this article is retained by the author(s), with first publication rights granted to the journal.

This is an open-access article distributed under the terms and conditions of the Creative Commons Attribution license (http://creativecommons.org/licenses/by/3.0/). 